\title{
Effects of feed rate and solution conductivity on jet current and fiber diameter in electrospinning of polyacrylonitrile solutions
}

\author{
Delaram Fallahi, ${ }^{1}$ Mehdi Rafizadeh, ${ }^{1 *}$ Naser Mohammadi, ${ }^{1}$ Behrooz Vahidi ${ }^{3}$ \\ ${ }^{1 *}$ Polymer Eng. Department, Amirkabir University of Technology, P.O.Box 15875- \\ 4413, Tehran, Iran; email: mehdi@aut.ac.ir \\ ${ }^{2}$ Iran Polymer and Petrochemical Institute, P.O.Box 14965-115, Tehran, Iran. \\ ${ }^{3}$ Electrical Eng. Department, Amirkabir University of Technology, P.O.Box 15875- \\ 4413, Tehran, Iran
}

(Received: 19 September, 2008; published: 8 September, 2009)

\begin{abstract}
Electrospinning of solutions of polyacrylonitrile in dimethylformamide (PAN/DMF) were performed under different voltages at different constant feed rates and the jet electric current was measured. The effects of applied voltage, feed rate and solution conductivity on jet electric current and fiber diameter were investigated. The results showed that the feed rate had no effect on jet current and fiber diameter, whereas by increasing the applied voltage and solution conductivity, both the jet current and fiber diameter were increased.
\end{abstract}

\section{Introduction}

Electrospinning is an effective process to produce sub-micron polymer fibers. The process is easy to implement. A syringe having a stainless steel needle is filled with a polymer solution. The solution is fed out at a constant flow rate forming a drop at the needle tip. An electrode of a high voltage power supply is connected to the syringe needle while a grounded collector is placed at a distance of about $20 \mathrm{~cm}$ from the needle. As the voltage is increased, electrical charge is induced on the fluid surface which distorts the solution drop at the needle tip. Above a critical voltage a single jet is ejected from the drop and moves toward the grounded collector. The electrical forces elongate the jet thousands of times and lead to accelerated solidification of the fluid jet and the collection of solid fibers on the collector.

It is well known that a wide range of parameters affect the electrospun fiber properties. These parameters are usually divided in two groups: process parameters such as voltage and flow rate [1, 2] and tip to target distance [3, 4]; and solution parameters such as concentration [5, 6], surface tension and conductivity [7, 8].

Some mathematical models have been introduced for electrically driven jets in electrospinning [9], and it is accepted that the jet electric current plays an important role in the process behavior and strongly affects the morphology of the electrospun fibers [1, 2, 10-17]. Kim et al. [18] have established a relation between the average electric current and the quality of the fibers. They have shown there is an optimum average electric current for the electrospinning process that depends on the solution properties. Theron et al. [19] reported that the average electric current increased with the increase in the feed rate and voltage. In some papers, the electric current was measured in real-time during the electrospinning process and different jet regimes 
were determined $[11,20]$. They investigated the relation between the jet electric current and the morphology of the fibers. He et al. [14-16] have introduced an allometric scaling relationship between the current and flow rate and also between the current and the applied voltage.

In this paper, in a horizontally electrospinning set-up, the jet current was measured during the experiments. The effect of feed rate and solution conductivity on the jet current and fibers diameter were investigated.

\section{Results and discussion}

\section{Effect of feed rate}

A solution of $10 \mathrm{wt} \%$ PAN in DMF was electrospun at different feed rates and the jet current was measured as a function of applied voltage. At each applied voltage no significant voltage drop across the resistor was observed when no electrospun jet was present. In other words, there was no corona discharge during the experiments. This increased the precision of the results because displacement current and corona discharge could insert some errors in the current measurements [2]. Fig. 1 shows the jet current vs. applied voltage at different feed rates.

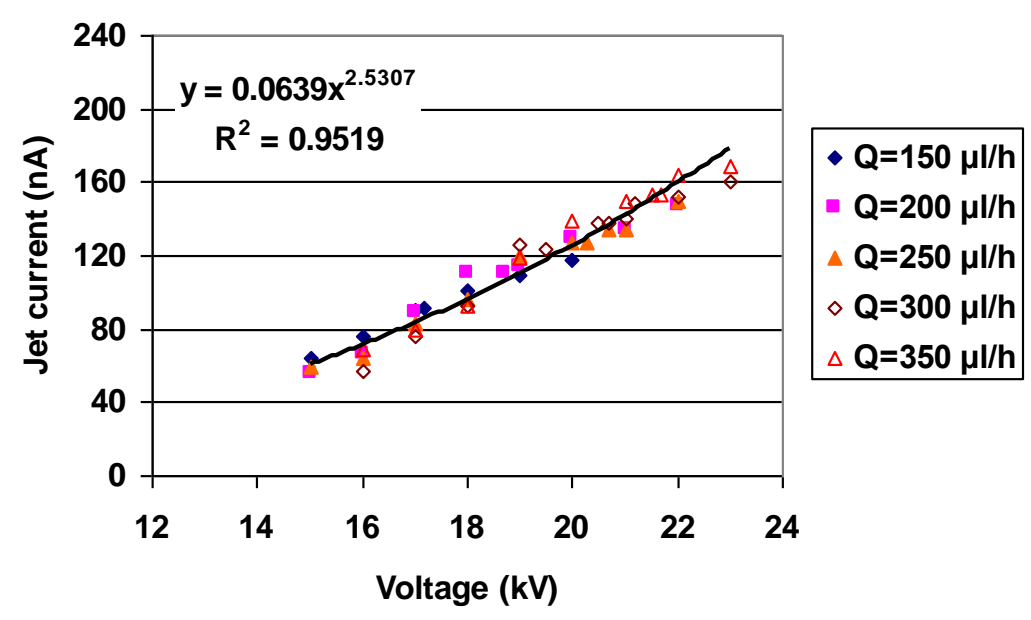

Fig. 1. Effect of applied voltage and feed rate on jet current in electrospinning of a 10 wt $\%$ PAN solution. The line shows the power-law curve-fitting to the data based on the least square method. The fitted equation and R-squared value are written on the chart.

As shown in Fig. 1, at all feed rates the jet current increased as the voltage was increased. In addition, it showed that the feed rate had no effect on the jet current and all the data lay on a single curve. There were some deviations in the results which could be attributed to random errors such as the variations in the applied voltage. In the high voltage power supply used in the experiments, the voltage adjustment system was analogous and little errors may have occurred in reading the voltage. Contrary to these results, in electrospinning of poly(ethylene oxide) solutions, an increase in the jet current by increasing the feed rate was reported elsewhere [1-2,19]. This difference may be explained by the direction of the process. In those reports $[1-2,19]$ the electrospinning process was done vertically, i.e. the jet 
moved downward, perpendicular to the ground. Since the jet streamed along the gravity, by increasing the feed rate the amount of the solution carried by the jet was also increased. In the present experiments the electrospinning process was done horizontally and the jet moved parallel to the ground. When the feed rate was increased, the drop of the solution at the needle tip grew large and dripped below the needle. So the feed rate did not affect the mass flow toward the collector and it had no effect on the jet current.

By power-law curve fitting on the data, the following relation between the jet current and the applied voltage was obtained:

$I=0.0639 V^{2.53}$

or:

current $\sim(\text { voltage })^{2.53}$

Similar relations with exponents of 2.7 and 2.8-3.1 were reported for solutions of polyurethane in DMF [5] and PAN in DMF [13] and polycaprolactone in (methylene chloride)/DMF[8], respectively. Shin et al. [2] has reported the linear increase of the current with the applied voltage, at low feed rates, for solutions of poly(ethylene oxide) in water.

To study the effect of the feed rate on fiber diameter, the SEM images of the fibers electrospun at a fixed voltage of $20 \mathrm{kV}$ and different feed rates were analyzed. As shown in Fig. 2 the average fiber diameter were practically and statistically unchanged with the changes in the feed rate. All of the $p$-values were higher than 0.05 , showing that there were no meaningful differences between the fiber diameters. The results showed that in a horizontally electrospinning set-up, the feed rate had no significant effect on the process.

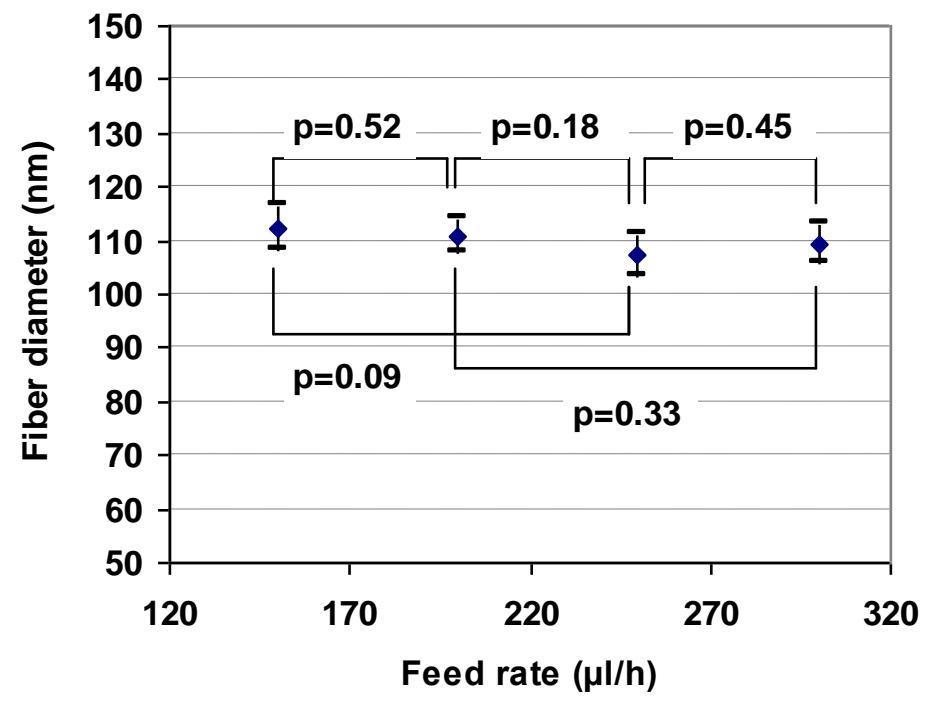

Fig. 2. The diameter of fibers as a function of feed rate. The process was done at a constant voltage of $20 \mathrm{kV}$. P-values were calculated using student t-test. 
There was no difference in the morphology of fibers as well. Fig. 3 shows the SEM images of fibers electrospun at a constant applied voltage and different feed rates. As shown in Fig. 3, the fibers produced at different feed rates had similar morphologies. In other words, it could be concluded that in the horizontally electrospinning set up used in the present research, the feed rate affected neither the jet current nor the fibers diameter and morphology.

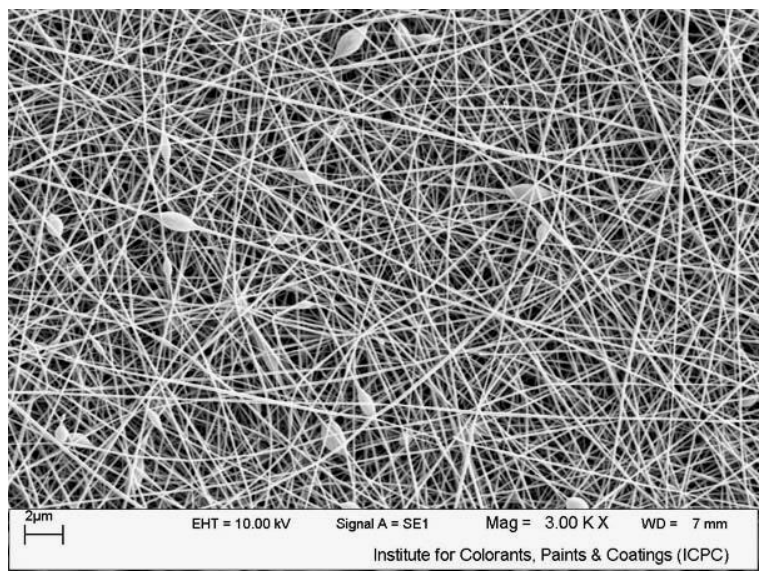

a

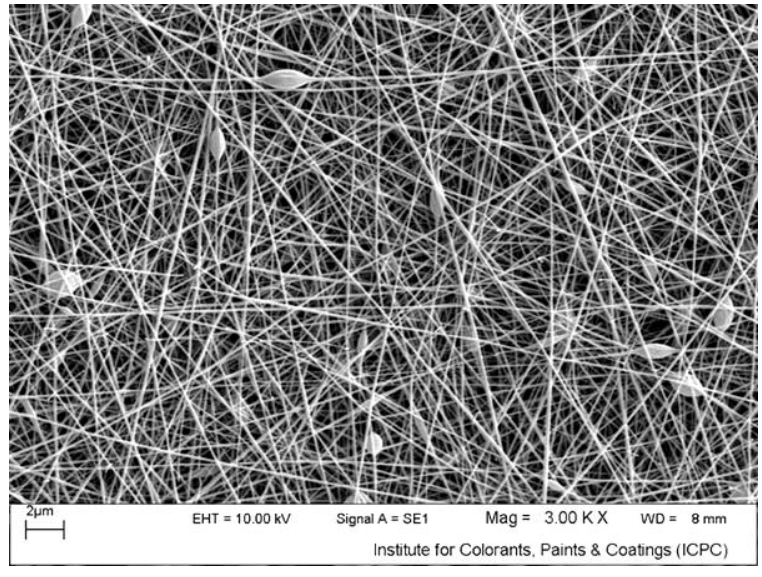

C

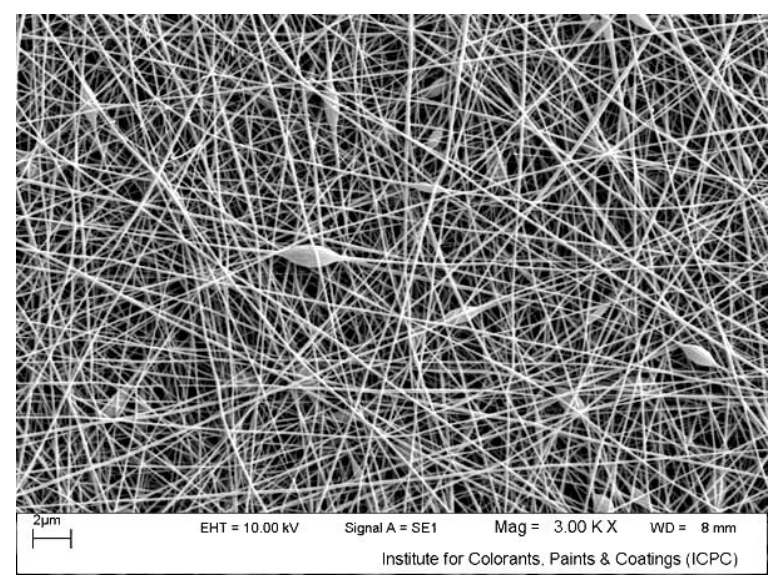

b

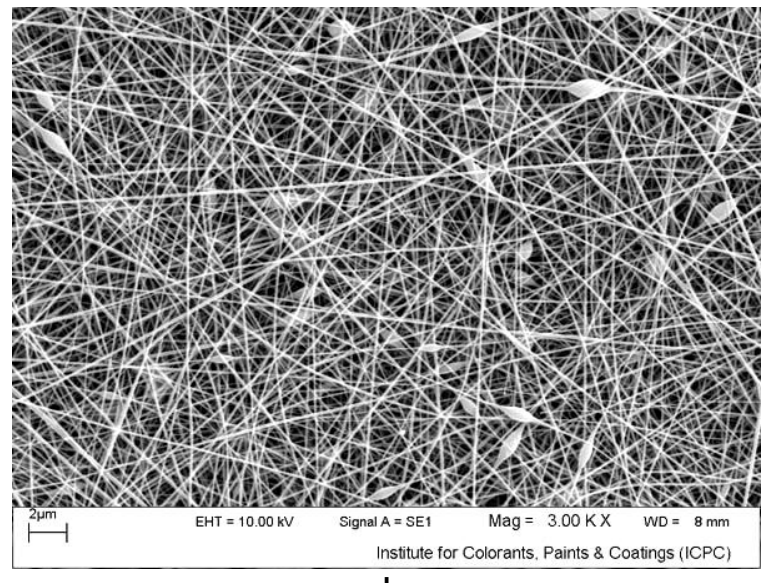

d

Fig. 3. SEM images of fibers obtained from electrospinning of a $10 \%$ PAN solution at a constant voltage of $20 \mathrm{kV}$ and feed rate of a) $150 \mu \mathrm{l} / \mathrm{h}$, b) $200 \mu \mathrm{l} / \mathrm{h}, \mathrm{c}) 250 \mu \mathrm{l} / \mathrm{h}, \mathrm{d}$ ) $300 \mu \mathrm{l} / \mathrm{h}$.

\section{Effect of solution conductivity}

To study the effect of solution conductivity on the jet electric current, a solution of 10 wt $\%$ PAN in DMF containing $0.1 \% \mathrm{LiCl}$ was electrospun at different feed rates and the jet current was measured as a function of applied voltage. Addition of $\mathrm{LiCl}$ to the solution increases the solution conductivity and results in changes in both the critical length of the straight jet and the fiber diameter [15, 21-22]. As shown in Fig. 4, similar to the previous section, the feed rate had no significant effect on the jet current. In comparison with Fig. 1, it is obvious that in the solution containing $\mathrm{LiCl}$, more electric charges were carried toward the collector. This could be attributed to higher conductivity of the solution. However, the relations between the applied voltage and the jet current in both solutions had almost the same exponents. Here, the relation between the applied voltage and the jet current was as: 
$I=0.0921 V^{2.57}$

or

current $\sim(\text { voltage })^{2.57}$

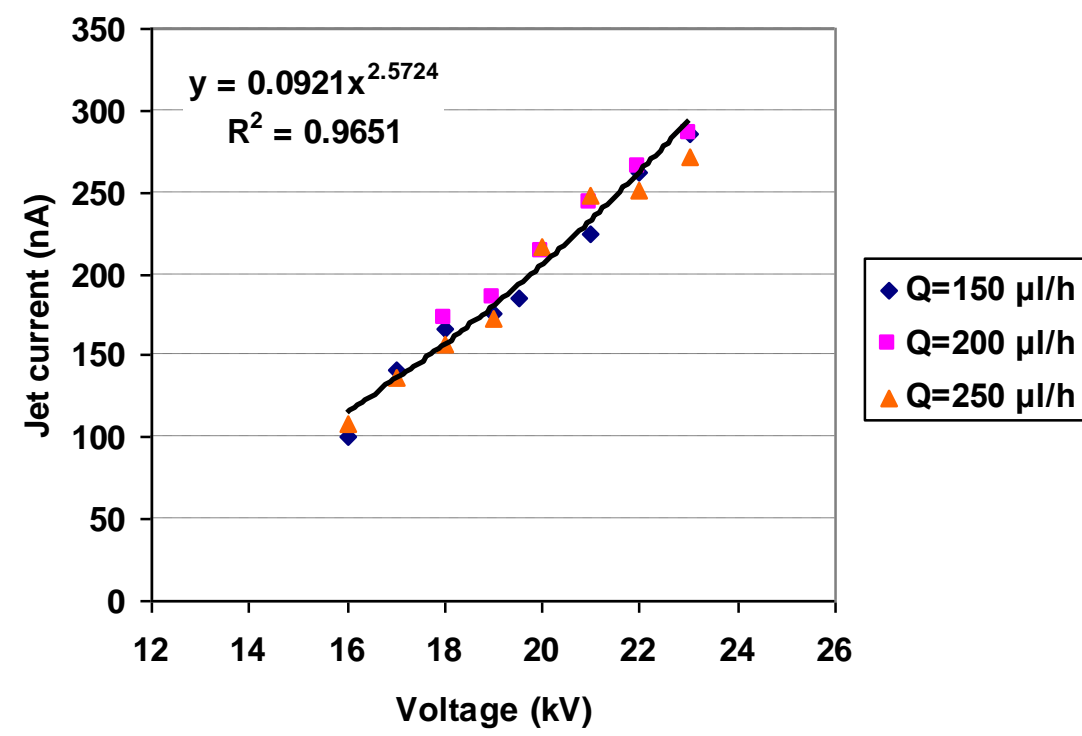

Fig. 4. Effect of applied voltage and feed rate on jet current in electrospinning of a 10 wt $\%$ PAN solution containing $0.1 \% \mathrm{LiCl}$. The line shows the power-law curve-fitting to the data based on the least square method. The fitted equation and R-squared value are written on the chart.

Fig. 5a shows the fiber diameter as a function of voltage for electrospinning of the mentioned solutions at different applied voltages. In electrospinning of both solutions, the fiber diameter was increased when the voltage was increased. The increase in fiber diameter as a function of the applied voltage was reported elsewhere too [5, 2325]. In addition, in Fig. $5 a$ it is obvious that fibers obtained from the solution with higher conductivity, had higher diameters. Increasing the voltage, as well as increasing the solution conductivity, raises the charge density of the jet. Increased charge density of the jet can affect the fiber diameter by two competing phenomena; a jet with higher net charge density is stretched with a stronger force which results in thinner fibers [7, 26-29]. On the other hand, because of the higher elongation force applied to the jet, more volume of the solution is carried toward the collector which produces fiber with higher diameter [5, 7, 23-25]. The observed increase or decrease in the fiber diameter depends on the dominant phenomenon.

Fig. 5b shows the fiber diameter as a function of the jet current. By increasing the jet current, the fiber diameter was increased. In this figure, the data obtained from solutions with different conductivities lie along each other, though they do not show exactly the same trend. Therefore, it is suggested that the jet current be used instead of the applied voltage to investigate the effect of solution conductivity on the fibers diameter. 


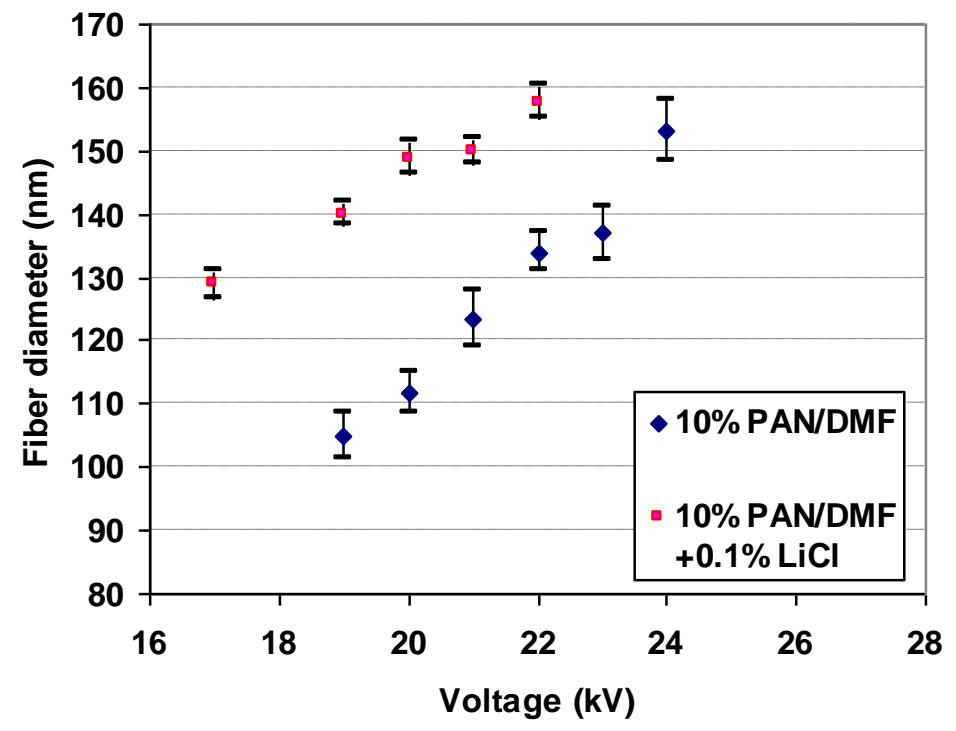

a

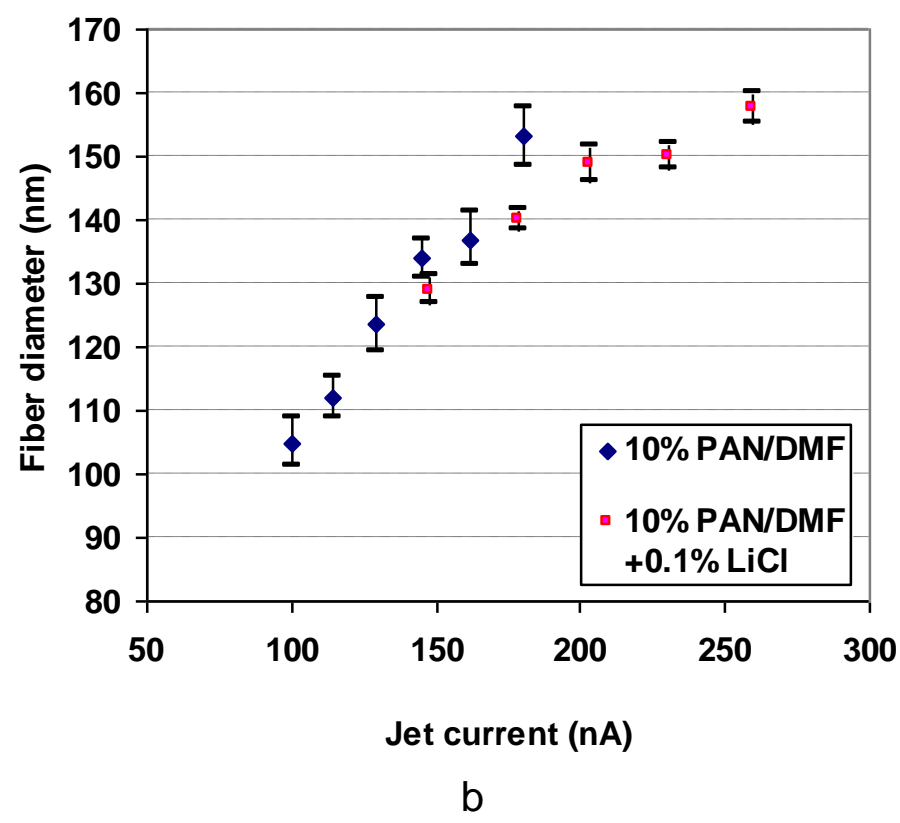

Fig. 5. Fiber diameter as a function of (a) voltage and (b) jet current.

\section{Conclusions}

Solutions of polyacrylonitrile in dimethylformamide (PAN/DMF) were electrospun in a horizontal electrospinning set-up. Different feed rates and voltages were applied and the jet electric current was measured during the process. The results showed that the jet current increased as a function of applied voltage and the feed rate had no effect on the jet current. The feed rate did not affect the fibers diameter and morphology either. Increasing the solution conductivity increased the jet current and the fiber diameter as well. 


\section{Experimental part}

Polyacrylonitrile (PAN) (Mv=80000 $\mathrm{g} / \mathrm{mol})$ was supplied by Iran Polyacryle Co. and dimethylformamide (DMF) and lithium chloride (LiCl) was purchased from Merck Co. A solution of 10 wt.\% PAN in DMF was prepared by constant mixing at room temperature for 2 hours. To increase the solution electrical conductivity, $0.1 \% \mathrm{LiCl}$ was added to the mixture at the beginning of the solution preparation.

The electrospinning set-up consisted of two aluminum disks (180 $\mathrm{mm}$ in diameter) that were placed vertically parallel to each other and $15 \mathrm{~cm}$ apart. A syringe (Hamilton, 80830) with a stainless steel needle $(I D=0.41 \mathrm{~mm})$ was fixed perpendicular to the center of the first disk while the needle protruded $18 \mathrm{~mm}$ from the surface of the disk. Both the needle and the first disk were connected to the positive electrode of a high voltage power supply. The second disk was used as the ground collector and was covered with a thin aluminum sheet at each run. The solution was fed at a constant volumetric flow rate using a syringe pump (New Era Pump System, NE1000). Jet current was measured as a voltage drop across a $10 \mathrm{M}$ resistor placed in series between the second disk and the ground. The voltage drop was sampled with a digital data acquisition system consisting of a PC (Pentium IV) and an Advantech $1711 \mathrm{l} / \mathrm{O}$ card with a sampling time of $10 \mathrm{~ms}$. The solutions were electrospun at room temperature, with feed rates of $150,200,250,300,350 \mu / / h$ while applying different voltages between 15 and $22 \mathrm{kV}$.

The morphology of electrospun fibers were observed on a scanning electron microscope (SEM) (Cambridge LEO 1455 VP). For each sample, the average diameter of the individual fibers was measured from multiple SEM images at the magnification of 15000 . The result for each sample was reported as an average value and confidence interval from at least 400 measurements. The data were statistically evaluated using student t-test and the p-values are reported [30].

\section{Acknowledgements}

The authors acknowledge the financial support of the Ministry of Science, Research and Technology of Iran.

\section{References}

[1] Reneker, D.H.; Yarin, L. Polymer 2008, 49, 2387.

[2] Shin, Y.M.; Hohman, M.M.; Brenner, M.P.; Rutledge, G.C. Polymer 2001, 42, 9955.

[3] Supaphol, P.; Chuangchote, S. J Appl Polym Sci 2008, 108, 969.

[4] Yang, Y.; Jia, Z.D.; Liu, J.N.; Wang, L.M.; Guan, Z.C. J Polym Eng 2008, 28, 67.

[5] Demir; M.M.; Yilgor, I.; Yilgor, E.; Erman, B. Polymer 2002, 43, 3303.

[6] He, J.H.; Wan, Y.Q.; Yu, J.Y. Fibers and Polymers 2008, 9, 140.

[7] Fallahi, D.; Rafizadeh, M.; Mohammadi, N.; Vahidi, B. e-polymers 2008, no. 56.

[8] Thompson, C.J.; Chase, G.G.; Yarin, A.L,; Reneker, D.H. Polymer 2007, 48, 6913.

[9] He, J.H.; Xu. L.; Wu, Y.; Liu, Y. Polym. Int. 2007, 56, 1323.

[10] Hohman, M.M.; Shin, M.; Rutledge, G.; Brenner, M.P. Phys Fluids 2001, 13, 2201.

[11] Samatham, R.; Kim, K.J.; Polym Eng Sci 2006, 46, 954.

[12] He, J. H.; Xu, L.; Wu, Y.; Liu, Y. Polym. Int. 2007, 56, 1323.

[13] Wan, Y.Q.; He, J.H.; Yu, J.Y. Iran Polym J 2006, 15, 265. 
[14] He, J.H.; Wan, Y.Q.; Yu, J.Y. Polymer 2005, 46, 2799.

[15] He, J.H.; Wu, Y.; Zuo, W.W. Polymer 2005, 4,6, 12637.

[16] He, J.H.; Wan, Y.Q.; Polymer, 2004, 45, 6731.

[17] Fridrikh, S.V.; Yu, J.H.; Brenner, M.P.; Rutledge, G.C. Phys Rev lett, 2003, 90,1445021 .

[18] Kim, S.J.; Shin, K.M.; Kim, S.I.; Scripta Mater., 2004, 51, 31.

[19] Theron, S.A.; Zussman, E.; Yarin, A.L. Polymer, 2004, 45, 2017.

[20] Fallahi, D.; Rafizadeh, M.; Mohammadi, N.; Vahidi, B. Polym. Int. to be published.

[21] Li, N. ; Qin, X.H. ; Yang, E.L.; Wang, S.U. Mater Lett 2008, 62,1345.

[22] Qin, X.H.; Wan, W.Q.; He, J.H.; Zhang, J.; Yu, J.Y.; Wang, S.Y. Polymer 2004, $45,6409$.

[23] Mit-uppatham, C.; Nithitanakul, M.; Supaphol P. Macromol. Chem. Phys. 2004, 205, 2327.

[24] Zong, X.; Kim, K.; Fang, D.; Ran, S.; Hsiao, B.S.; Chu, B. Polymer 2002, 43, 4403.

[25] Manee-in, J.; Nithitanakul, M.; Supaphol P. Iran. Polym. J. 2006, 15, 341.

[26] Buchko, C.J.; Chen, L.C.; Shen, Y.; Martin, D.C. Polymer 1999, 40, 7397.

[27] Megelski, S. Stephens, J.S.; Chase, D.B.; Rabolt, J.F. Macromolecules 2002, 35, 8456.

[28] Lee, K.H.; Kim, H.Y.; La, Y.M.; Lee, D.R.; Sung, N.H. J. Polym. Sci. B: Polym. Phys. 2002, 40, 2259.

[29] Jalili, R.; Hosseini, S.A.; Morshed, M. Iran. Polym. J. 2005, 14, 1074.

[30] Finner, H.; Strassburger, K. Biometrical J. 2007; 49: 941. 